evaluate its integration with the host brain. METHODS/STUDY POPULATION: To optimize cell density and health, silk fibroin scaffolds varying in porosity and stiffness were seeded with E18 GFP + rat cortical neurons and imaged at DIV 5. Different seeding methods and loads were similarly tested. Constructs, loaded with an inhibitor of apoptosis (ROCK inhibitor Y-27632) or necroptosis (necrostatin-1) in a fibrin hydrogel, were transplanted into aspiration lesions created in the primary motor cortex of SpragueDawley rats, and graft survival was compared to negative control at 2 weeks. Lastly, constructs were transplanted and evaluated via immunohistochemistry at 1, 2, and 4-month time points for survival, differentiation, inflammation, and anatomic integration. RESULTS/ ANTICIPATED RESULTS: Scaffolds with smaller pore sizes retained more cells after seeding. Softer scaffolds, which enhance hemostasis at transplantation, did not compromise cell health on live/dead assay. We anticipate that seeding concentrated cell suspensions onto multiple surfaces of the construct will produce the most evenly seeded and cell-dense constructs. Based on a prior pilot study, we anticipate that necrostatin- 1 will significantly improve intermediate-term construct survival. We have observed up to $15 \%$ cell survival at 1 month with retained neuronal identity and abundant axonal projections into the brain despite evidence of persistent inflammation; we anticipate similar outcomes at later time points. DISCUSSION/SIGNIFICANCE OF IMPACT: Our construct, due to its exceptional longevity in vitro, manipulability, and modularity, is an attractive platform for neural tissue engineering. In the present work, we optimize and validate this technology for transplantation with the goal of addressing the morbidity burden of cortical injury.

4303

\section{Optimization of primary septal-hippocampal co-cultures to study central cholinergic synapse formation and dysfunction}

Sarra Djemil ${ }^{1}$, Claire R. Ressel, and Daniel T.S. Pak

${ }^{1}$ Georgetown - Howard Universities

OBJECTIVES/GOALS: Septal cholinergic innervation to the hippocampus is critical for normal learning and memory and is severely degenerated in Alzheimer's disease. To understand the molecular events underlying this loss, we optimized a primary septal-hippocampal co-culture system that facilitates study of central cholinergic synapses. METHODS/STUDY POPULATION: We developed an optimized in vitro septal-hippocampal co-culture system modified from previous published protocols. Briefly, hippocampal and septal tissue were harvested from embryonic day 19 (E19) SpragueDawley rats, digested with $0.1 \%$ trypsin, and an equal number of cells from each region plated onto coverslips coated with poly-D-lysine and laminin at a final density of 300 cells $/ \mathrm{mm}^{2}$. We use immunostaining with validated primary antibodies and a fluorescent binding assay, together with confocal microscopy, to determine the structure of cholinergic synapses that are 1) native, 2) mammalian, 3) CNS derived, 4) comprised of physiological synaptic partners, and 5) developmentally mature. RESULTS/ANTICIPATED RESULTS: After DIV21, co-cultures maintained a healthy morphology. A subpopulation of neurons strongly expressed the cholinergic markers vesicular ACh transporter (vAChT), choline acetyltransferase (ChAT), and the high-affinity choline transporter (ChT1), whereas most neurons lacked vAChT expression and were presumably glutamatergic or GABAergic. The percentage of cholinergic neurons in the co-culture attained up to $\sim 5-7 \%$, depending on conditions such as embryo age at dissection or ratio of septal to hippocampal cells. We also report on cholinergic synapse structure by examining postsynaptic markers (excitatory and inhibitory) and staining for nicotinic acetylcholine receptor subunits. DISCUSSION/SIGNIFICANCE OF IMPACT: Primary septal-hippocampal co-cultured neurons have not been exploited extensively in the field, perhaps due to the difficulty in maintaining such cultures for extended periods. Here, we optimized an in vitro septal-hippocampal co-culture system, a powerful tool to comprehensively analyze central cholinergic synapse formation and dysfunction.

4072

Optimizing ex-vivo perfusion in Vascularized Composite Allotransplantation using Hyperosmolar solution and Electric Stimulation: Preliminary Results

Michael Jonczyk ${ }^{1}$, and Philipp Tratnig-Frankl ${ }^{2}$

${ }^{1}$ Tufts University; ${ }^{2}$ Division of Plastic and Reconstructive Surgery, MGH

OBJECTIVES/GOALS: Vascularized composite allotransplantation (VCA) restores devastating soft tissue injuries. However, graft viability may be compromised during ischemia time, thus preservation techniques continue to evolve. Here we summarize our preliminary findings from preservation techniques utilizing hyperosmolar extracellular solution (HES) and electric stimulation in a 6 hour ex-vivo perfusion model. METHODS/STUDY POPULATION: A published, MGH rodent hindlimb ex-vivo perfusion model was utilized for this project. Three baseline control elements were taken to compare our results including: a baseline muscle biopsy, harvested hindlimb preserved on ice $\left(4^{\circ} \mathrm{C}\right)$ in static cold storage (SCS), and 6 hour perfusion (SNMP) were used to compare results of our four aims. The four aims are shown in Table 1. HES was composed of muscle media and the addition of mannitol until 3 concentrations were made: 300, 500, and 800 mOsm concentrations. In aim 4, the perfusate composition was changed to test a hyper-oncotic purfusate. After 6 hours a muscle biopsy was taken to analyze energy cofactors via liquid chromatography-mass spectrometry, referred to as energy charge. Weight gain (edema), lactate levels, oxygen consumption and energy charge (EC) were used as markers for muscle tissue viability. RESULTS/ANTICIPATED RESULTS: In Aim 1, the higher osmolarity of HES indirectly reduced weight gain but consequentially reduced the EC below 5\% when compared to SCS control group. We next incorporate HES into the perfusion model, Aim 2, and noticed a diminution in weight gain. The 500 mOsm group had substantial improvement in EC, lactate production and improved oxygen exchange when compared to a controls: fresh muscle biopsy and SNMP. In Aim 3, after a 6 hour perfusion with the addition of electric stimulation, graft edema improved by $10 \%$, EC improved by $23 \%$ and $\mathrm{O} 2$ dissociation was highest of all 4 aims. Consequentially, due to muscular contraction the lactate levels were highest. In Aim 4, using a hyper-oncotic perfusate, edema reduced the most during the 6 hour perfusion but revealed lower EC and similar lactate/O2 results. However when left on for 24 hours, edema was significantly higher with lactate build up, EC improved with time as well as $\mathrm{O} 2$ dissociation. DISCUSSION/SIGNIFICANCE OF IMPACT: Here we have shown our preliminary results comparing our known ex-vivo perfusion model to multiple hypothesis to improve VCA graft viability. These preservation techniques demonstrate promising results but further studies are ongoing to confirm this encouraging outcome. 